\title{
Suppression of SIRT6 by miR-33a facilitates tumor growth of glioma through apoptosis and oxidative stress resistance
}

\author{
MINGZE CHANG ${ }^{1,2 *}$, LIN QIAO $^{3 *}$, BIN LI $^{1}$, JUANHONG WANG $^{4}$, GEJUAN ZHANG $^{2}$, WENZHEN SHI $^{1}$, \\ ZHIQIN LIU ${ }^{1}$, NAIBING GU ${ }^{1}$, ZHENGLI DI $^{1}$, XINLAI WANG $^{1}$ and YE TIAN ${ }^{2}$ \\ ${ }^{1}$ Department of Neurology, Xi'an Central Hospital, Xi'an Jiaotong University School of Medicine, Xi'an, Shaanxi 710003; \\ ${ }^{2}$ Department of Neurology, Xi'an Third Hospital, Xi'an, Shaanxi 710004; ${ }^{3}$ Department of Anesthesiology, \\ Xi'an No. 5 Hospital, Xi'an, Shaanxi 710082; ${ }^{4}$ Department of Pathology, Xi'an Central Hospital, \\ Xi'an Jiaotong University School of Medicine, Xi'an, Shaanxi 710003, P.R. China
}

Received December 12, 2016; Accepted June 23, 2017

DOI: $10.3892 / o r .2017 .5780$

\begin{abstract}
RNA-33a (miR-33a) belongs to the miR-33 family that is implicated in the progression of various types of cancers. Aberrant expression of miR-33a has been detected in several human cancers, and has been shown to regulate the migration and invasion as well as proliferation and apoptosis of tumor cells. However, the clinical significance and precise mechanisms underlying the dysfunction of miR-33a in glioma have not been well investigated in previous studies. In this study, overexpression of miR-33a was observed in clinical glioma specimens and cell lines. Clinicopathological detection revealed that miR-33a highly expressing patients showed large tumor sized and advanced World Health Organization (WHO) grade as well as reduced overall survival. Furthermore, the results of in vitro experiments confirmed that loss of miR-33a resulted in reduced proliferation and enhanced apoptosis in U251 cells, while miR-33a restoration showed opposite effects in U87 cells. Further studies indicated that miR-33a knockdown restrained tumor growth of glioma in vivo. miR-33a negatively regulated the expression of sirtuin 6 (SIRT6) at both mRNA and protein levels via targeting the 3'UTR of SIRT6 mRNA. SIRT6 was underexpressed and inversely correlated with miR-33a expression in the glioma tissues. Mechanistically, SIRT6 overexpression increased the levels of lactate dehydrogenase (LDH) and reactive oxygen species (ROS) while it reduced cell survival under $\mathrm{H}_{2} \mathrm{O}_{2}$ treatment. In addition, SIRT6 restoration led to apoptosis with alterative expression of Bax, Bcl-2, cleaved caspase-8, and inhibition of Janus kinase 2 (JAK2)/signal transducer and activator of transcription 3 (STAT3) pathway in glioma. Thus, our studies
\end{abstract}

Correspondence to: Professor Ye Tian, Department of Neurology, Xi'an Third Hospital, Xi'an, Shaanxi 710004, P.R. China

E-mail: tianye_xian@163.com

${ }^{*}$ Contributed equally

Key words: glioma, miR-33a, SIRT6, tumor growth, apoptosis, oxidative stress demonstrated that the deregulation of miR-33a may promote tumor development in human glioma by regulating the expression of its target gene, SIRT6.

\section{Introduction}

Glioma is the most common, aggressive and lethal type of malignant tumor in the central nervous system (1). Its relapse and mortality rate are increasing due to the inefficiency of current treatment leading to a poor prognosis despite the combination of multidisciplinary therapies including surgical resection, chemotherapy and radiotherapy $(2,3)$. Despite the diverse therapeutic efforts, the median survival of glioma patients has not obviously changed (4). The poor prognosis highlights the urgent requirement for elucidating the detailed molecular mechanisms for the development of novel therapeutic tools against glioma and the identification of diagnostic and prognostic markers of glioma (5).

As a class of small non-coding RNAs, microRNAs (miRNAs) target the 3'UTR regions of multiple mRNAs and block translational process, which leads to gene silence (6). One of the gene clusters targeted by miRNAs has been confirmed to be associated with tumor growth and aggressiveness (7). Accumulating evidence indicates that miR-33a functions as either an oncogene or tumor suppressor in various types of human cancer. miR-33a was found to function as a tumor suppressor and to inhibit tumor growth by repressing Pim-1 (8). Furthermore, miR-33a regulation by Twist1 restrained epithelial-to-mesenchymal transition and metastasis in non-small-cell lung carcinoma (NSCLC) (9). Previous research indicates that miR-33a expression is inhibited in various cancer types including NSCLC, gallbladder cancer, hepatocellular carcinoma (HCC), breast cancer, melanoma and pancreatic cancer (9-16). However, overexpression of miR-33a has been reported in prostate cancer (17). Furthermore, miR-33a was found to contribute to osteosarcoma chemoresistant by reducing cisplatin-induced cell apoptosis (18). The high expression of miR-33a has been previously detected in glioblastoma specimens and is significantly correlated with a poor prognosis (19). miR-33a facilitates the malignant behaviors of glioma-initiating cells including growth and self-renewal 
by targeting protein kinase A (PKA) and Notch signaling pathways (19). However, the clinical significance and precise mechanisms underlying the dysfunction of miR-33a in glioma have not been well investigated in previous studies.

In this study, miR-33a was revealed as an upstream regulator of sirtuin 6 (SIRT6) which reversed the malignant potential and reactive oxygen species (ROS) resistance of glioma. High levels of miR-33a accompanied by decreased SIRT6 expression were detected in glioma cells and tissues, compared with these levels in normal cells and tissues. Further analysis suggested that restoration of SIRT6 in glioma cells resulted in reduced cell survival, oxidative stress-induced apoptosis as well as repression of Janus kinase 2 (JAK2)/signal transducer and activator of transcription 3 (STAT3) pathways. Therefore, our study demonstrated that aberrant overexpression of miR-33a disrupts the inhibitory effect of SITR6 and consequently promotes the malignant phenotypes of glioma.

\section{Materials and methods}

Patients. Glioma specimens and the corresponding normal tissues were acquired from 60 glioma patients who underwent surgery at Xi'an Central Hospital, Xi'an Jiaotong University School of Medicine. Patients who received immunotherapy, chemotherapy or radiotherapy before surgical treatment were excluded. Signed informed consent was provided by each patient before clinical specimens were collected and used. Tissue specimens were conserved in liquid nitrogen or $10 \%$ formalin until use. All clinicopathological information of the glioma patients is documented in Table I. The study was performed following the approval of the Ethics Committee of the Xi'an Jiaotong University School of Medicine.

Cell culture and reagents. Human glioma cell lines, U87, T98, A172 and U251, and a normal human astrocyte (NHA) cell line were purchased from the Cell Bank of Shanghai Institute of Cell Biology (Chinese Academy of Medical Science, Shanghai, China) and cultured in Dulbecco's modified Eagle's medium (DMEM) supplemented with 10\% fetal bovine serum (FBS) (both from Gibco, Grand Island, NY, USA) at $37^{\circ} \mathrm{C}$ with $5 \% \mathrm{CO}_{2}$.

miR-33a mimic and inhibitor as well as their corresponding negative control (NC) vectors were purchased from GenePharma (Shanghai, China). pcDNA3.1-SIRT6 and the empty vector were obtained from Shanghai Genechem Co., Ltd., (Shanghai, China). Vectors were transferred into cells using Lipofectamine 2000 (Invitrogen, Carlsbad, CA, USA) on the basis of the manufacturer's recommendation. $\mathrm{H}_{2} \mathrm{O}_{2}(30 \%)$ was purchased from Sigma-Aldrich (St. Louis, MO, USA).

Immunohistochemistry. All specimens were fixed in $10 \%$ neutral formalin, embedded in paraffin and cut into $4-\mu \mathrm{m}$ sections for immunohistochemical staining. The EnVision ${ }^{\mathrm{TM}}$ two-step method was used (Dako, Hamburg, Germany), as well as the following antibody: SIRT6 primary antibody (Abcam, Cambridge, MA, USA).

Immunoblotting. Cells were dissociated in RIPA lysis buffer (P0013D; Beyotime, Haimen, China) and PMSF (ST506) (Beyotime). A Bradford protein assay kit (P0006; Beyotime)
Table I. Correlation between the clinicopathological characteristics of the glioma cases and miR-33a expression.

\begin{tabular}{|c|c|c|c|c|}
\hline \multirow[b]{2}{*}{ Characteristics } & \multirow[b]{2}{*}{$\begin{array}{l}\text { Total } \\
\mathrm{n}=60\end{array}$} & \multicolumn{2}{|c|}{ miR-33a expression } & \multirow[b]{2}{*}{ P-value } \\
\hline & & $\begin{array}{c}\text { High } \\
(\mathrm{n}=30)\end{array}$ & $\begin{array}{c}\text { Low } \\
(\mathrm{n}=30)\end{array}$ & \\
\hline \multicolumn{5}{|l|}{ Age (years) } \\
\hline$<50$ & 28 & 12 & 16 & \multirow[t]{2}{*}{0.301} \\
\hline$\geq 50$ & 32 & 18 & 14 & \\
\hline \multicolumn{5}{|l|}{ Sex } \\
\hline Male & 33 & 15 & 18 & \multirow[t]{2}{*}{0.436} \\
\hline Female & 27 & 15 & 12 & \\
\hline \multicolumn{5}{|l|}{ Tumor size $(\mathrm{cm})$} \\
\hline$<5$ & 22 & 7 & 15 & \multirow[t]{2}{*}{$0.032^{\mathrm{a}}$} \\
\hline$\geq 5$ & 38 & 23 & 15 & \\
\hline \multicolumn{5}{|l|}{ KPS score } \\
\hline$<80$ & 34 & 14 & 20 & \multirow[t]{2}{*}{0.118} \\
\hline$\geq 80$ & 26 & 16 & 10 & \\
\hline \multicolumn{5}{|l|}{ WHO grade } \\
\hline I+II & 20 & 6 & 14 & \multirow[t]{2}{*}{$0.028^{\mathrm{a}}$} \\
\hline III+IV & 40 & 24 & 16 & \\
\hline
\end{tabular}

WHO, World Health Organization; KPS, Karnofsky performance

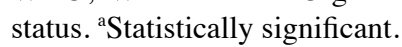

was used to analyzed protein concentrations, and the proteins were loaded for $10 \%$ SDS-PAGE electrophoresis, and then the proteins after separation were transferred onto PVDF membranes (Sigma). Then the PVDF membranes were blocked with 5\% skim milk (Guangming, Shanghai, China) and incubated with the primary antibody at $4^{\circ} \mathrm{C}$ overnight. Then, specimens were incubated with a secondary antibody conjugated with HRP (Cell Signaling Technology, Beverly, MA, USA). $\beta$-actin (Santa Cruz Biotechnology, Santa Cruz, CA, USA) was used as a loading control. SIRT6 primary antibody was obtained from Abcam. Bax, Bcl-2, caspase-8, p-JAK2, JAK2, p-STAT3 and STAT3 primary antibodies were purchased from Cell Signaling Technology.

RNA extraction and quantitative PCR. qRT-PCR was carried out as previously described (9). Total RNA was isolated from clinical tissue samples with a Total RNA isolation kit (AP-MNMS-RNA; Axygen, Union City, CA, USA) as described by the manufacturer. Total RNA from cells was isolated with TRIzol reagent (Invitrogen) and miRNAs were isolated using a microRNA purification kit (Norgen Biotek, Thorold Ontario, Canada), according to the manufacturer's protocol. miRNAspecific quantitative PCR was performed with Taqman microRNA assay primers (Applied Biosystems, Foster City, CA, USA) according to the manufacturer's instructions. The levels of miRNAs expression were normalized by U6 RNA. qPCR for SIRT6 mRNA was performed with SYBR-Green PCR Master Mix (Applied Biosystems). $\beta$-actin was employed as the internal control. The primers were synthesized and purchased from Sangon Biotech (Shanghai, China). 

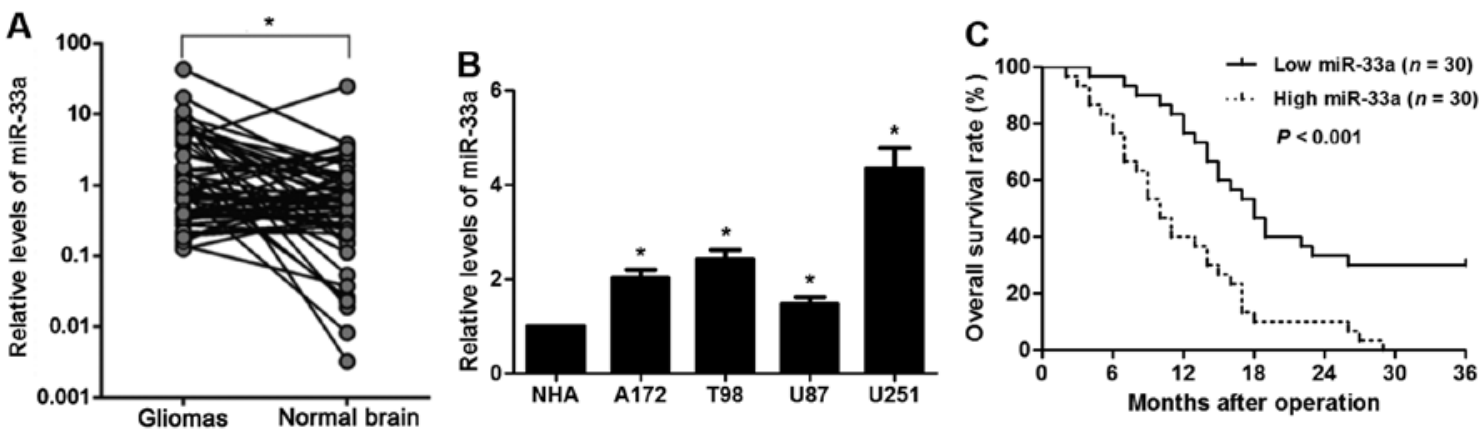

Figure 1. miR-33a shows increased expression in gliomas. (A) qRT-PCR was performed to identify the relative expression of miR-33a in 60 paired primary glioma samples. t-test was used for statistical analysis. "P<0.05. (B) The expression of miR-33a in glioma cell lines (A172, T98, U87 and U251) compared with NHA cells. t-test was used for statistical analysis. "P<0.05. (C) Kaplan-Meier analysis demonstrated that patients with high expression of miR-33a showed a notably reduced overall survival compared to miR-33a low expressing cases. NHA, normal human astrocytes.

Colony formation assay. In regards to the colony formation assay, 2,000 glioma cells were seeded on 6-well plates. Fourteen to twenty-one days after cell seeding, cell colonies with crystal violet staining were counted.

Cell cytotoxicity assay. The indicated cells were seeded on 96-well plates and cultured for $24 \mathrm{~h}$ followed by $\mathrm{H}_{2} \mathrm{O}_{2}$ at different concentrations for $24 \mathrm{~h}$. CCK-8 cell viability and lactate dehydrogenase (LDH) assay were performed as previously reported (20). ROS levels were measured as previously described (21).

Flow cytometric detection of apoptosis. The indicated cells were collected for detection of apoptosis using the Annexin V FLUOS kit (Roche, Indianapolis, IN, USA) according to the manufacturer's instructions. All of the samples were assayed in triplicate.

Experimental animals. Six-week-old male athymic BALB/c nude mice were subcutaneously injected with $2 \times 10^{6} \mathrm{U} 251$ cells with NC inhibitor or miR-33a inhibitor transfection $(n=6$, respectively). The tumor volumes were calculated using the standard formula: tumor volume $\left(\mathrm{mm}^{3}\right)=$ longer diameter $\mathrm{x}$ (shorter diameter) ${ }^{2} / 2$. The in vivo study was approved by the Institutional Animal Care and Use Committee of Xi'an Jiaotong University School of Medicine.

Luciferase reporter assay. Wild-type (wt) or mutant (mt) 3'UTR of SIRT6 was amplified and cloned into pmiR-RB-REPORT ${ }^{\mathrm{TM}}$ luciferase. Luciferase reporter containing the potential binding sequence of 3'UTR of SIRT6 was co-transfected with miR-33a mimic or negative control mimic in U87 cells in a 96-well plate. Two days later, dual-luciferase reporter assay system (Promega, Madison, WI, USA) was used to measure the alteration of luciferase. Firefly luciferase activity was normalized to Renilla luciferase activity.

Statistical analysis. All statistical analyses were carried out using GraphPad Prism 5 software (GraphPad Software, Inc., San Diego, CA, USA). Experimental data are presented as mean \pm SEM from at least three independent experiments. The data were analyzed by the Chi-squared test, Student's t-test, ANOVA, log-rank test and Pearson's correlation test. A p-value $<0.05$ was considered statistically significant.

\section{Results}

Upregulation of miR-33a is observed in clinical glioma tissues. Consistent with the dysregulation of miR-33a in other tumor types, the levels of miR-33a were overexpressed in the glioma samples when compared with levels in the normal tissues ( $\mathrm{P}<0.05$, Fig. 1A). In addition, the expression of miR-33a in glioma cell lines (U87, T98, A172 and U251) was higher than that in a NHA cell line $(\mathrm{P}<0.05$, respectively, Fig. 1B). Then we defined the cutoff value between the miR-33a low and high expression group as the median expression level of miR-33a in glioma. Further analysis of the clinical specimens showed that high expression of miR-33a was positively correlated with large tumor size and advanced World Health Organization (WHO) grade $(\mathrm{P}<0.05$, respectively) but not with age, sex and Karnofsky performance status (KPS) score (Table I). In addition, the 3-year overall survival (OS) rate of the miR-33a high expression group was significantly lower than that of the miR-33a low expression group $(\mathrm{P}<0.001$, Fig. 1C). These data suggest that overexpression of miR-33a indicates a poor prognosis for glioma patients.

$m i R-33$ a regulates the proliferation and apoptosis of glioma cells. The roles of miR-33a in cancer progression and metastasis have been discussed in different types of tumor. Thus, we aimed to elucidate whether silencing of miR-33a prohibits the malignant phenotypes of glioma. miR-33a inhibitor transfection resulted in obvious downregulation of miR-33a in the U251 cells ( $\mathrm{P}<0.05$, Fig. $2 \mathrm{~A}$ ). The results of CCK- 8 and colony formation assays showed that $\mathrm{U} 251$ cells transfected with the miR-33a inhibitor presented a reduced cell proliferation, compared with the control cells $(\mathrm{P}<0.05$, respectively, Fig. $2 \mathrm{~B}$ and $\mathrm{C})$. In addition, stable knockdown of miR-33a induced apoptosis of the U251 cells $(\mathrm{P}<0.05$, Fig. 2D). In contrast, the expression of miR-33a was restored by miR-33a mimic in the U87 cells ( $\mathrm{P}<0.05$, Fig. 3A). Notably, miR-33a overexpression enhanced $\mathrm{U} 87$ cell growth and reduced apoptosis in vitro $(\mathrm{P}<0.05$, respectively, Fig. 3B-D). Next, a subcutaneous implantation model of human glioma was established in nude mice. Tumor growth curves and tumor weight revealed that the miR-33a inhibitor resulted in reduced in vivo growth of glioma cells compared with the control cells $(\mathrm{P}<0.05$, respectively, Fig. 4A-C). These data indicate that miR-33a influences the proliferative and apoptotic capabilities of glioma cells. 

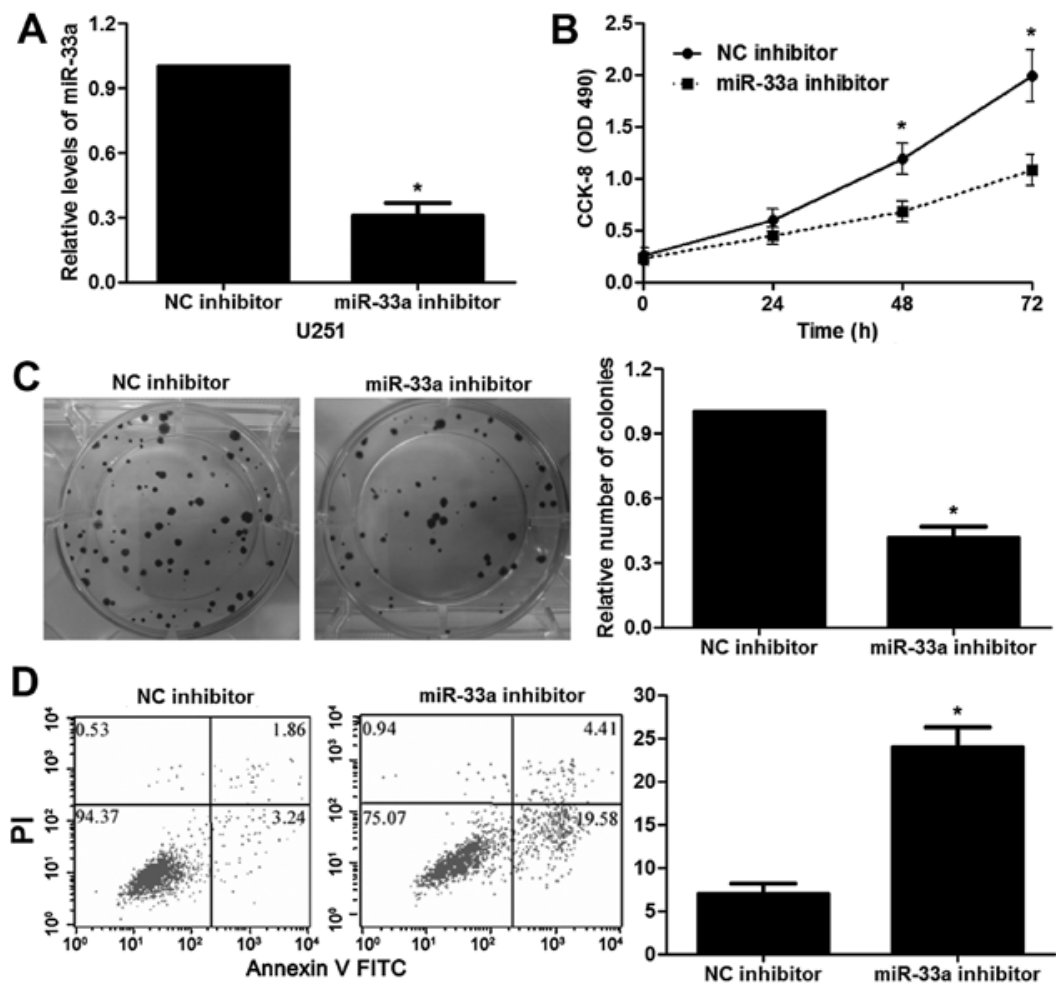

Figure 2. Loss of miR-33a inhibits proliferation and induces apoptosis in U251 cells. (A) U251 cells that were transfected with miR-33a inhibitor and negative control (NC) inhibitor, respectively, were subjected to qRT-PCR for miR-33a expression. t-test was used for statistical analysis. "P<0.05. (B) MTT assays revealed that miR-33a knockdown inhibited cell proliferation in the U251 cells. ANOVA analysis was used for statistical analysis. "P<0.05. (C) The number of colonies were reduced after miR-33a knockdown in the U251 cells. t-test was used for statistical analysis. ${ }^{*} \mathrm{P}<0.05$. (D) The apoptosis of U251 cells was increased as measured by flow cytometry after miR-33a silencing. t-test was used for statistical analysis. ${ }^{*} \mathrm{P}<0.05$.
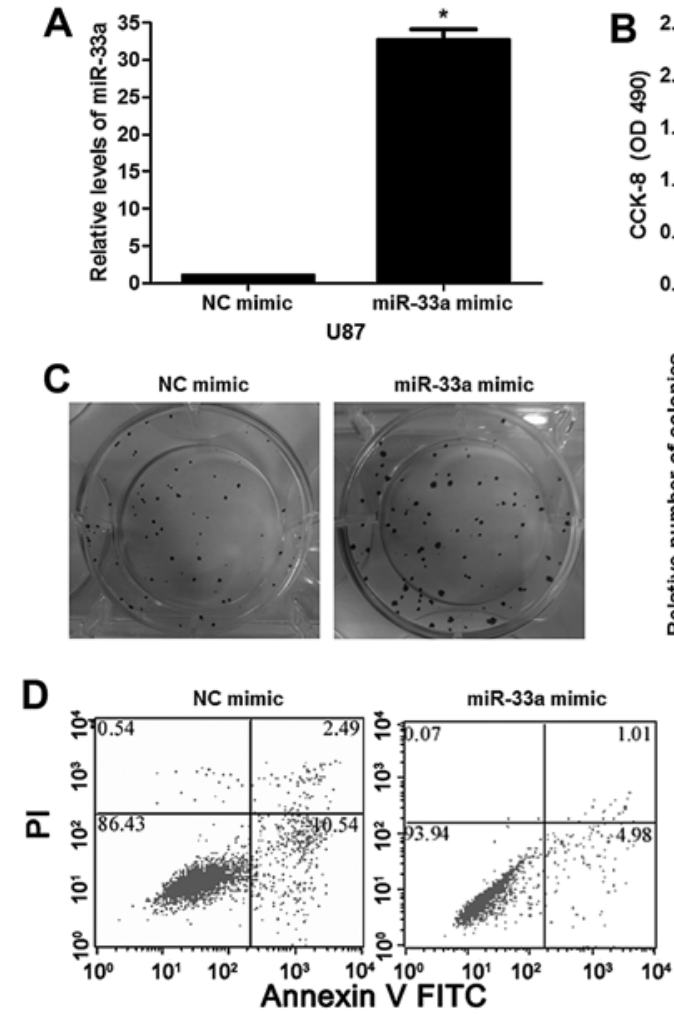
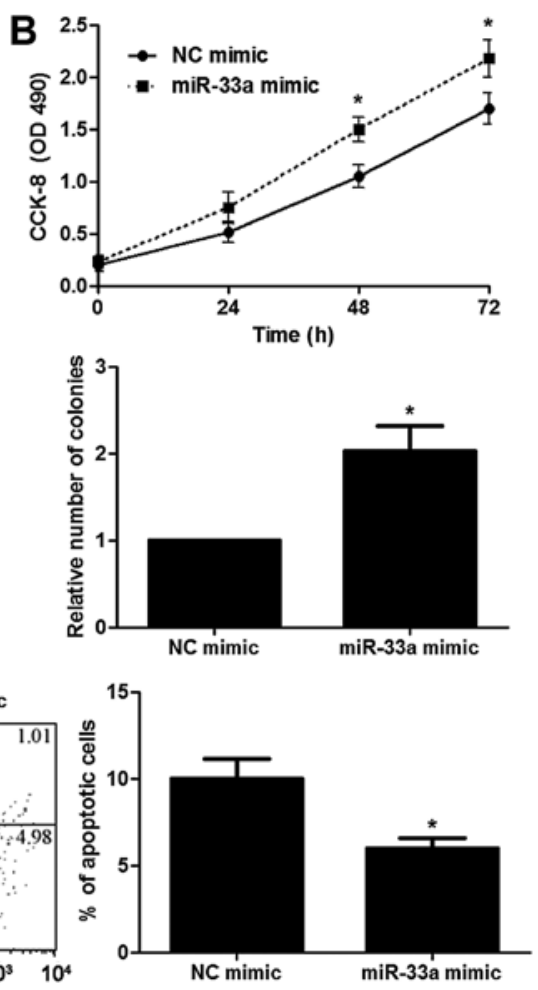

Figure 3. miR-33a overexpression leads to enhanced proliferation and decreased apoptosis in the U87 cells. (A) U87 cells that were transfected with miR-33a mimic and negative control (NC) mimic, respectively, were subjected to qRT-PCR for miR-33a expression. $t$-test was used for statistical analysis. "P<0.05. (B) MTT assays revealed that miR-33a overexpression facilitated cell proliferation in the U87 cells. ANOVA test was used for statistical analysis. "P<0.05. (C) The number of colonies were increased after miR-33a overexpression in the U87 cells. t-test was used for statistical analysis. "P<0.05. (D) The apoptosis of U87 cells was decreased as measured by flow cytometry after miR-33a overexpression. t-test was used for statistical analysis. "P<0.05. 
A

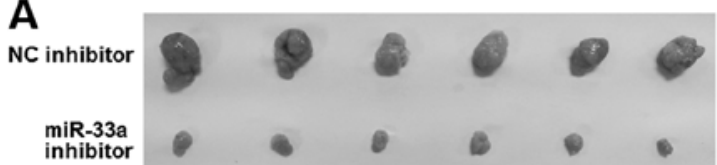

B
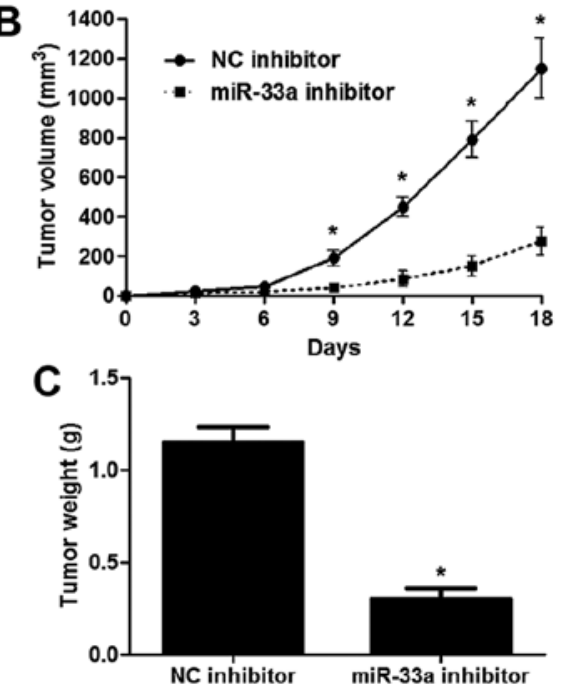

Figure 4. miR-33a knockdown inhibits tumor formation of glioma in nude mice. (A) U251 cells that were transfected with miR-33a inhibitor and negative control (NC) inhibitor, respectively, were implanted into nude mice via subcutaneous injection. Tumors were collected from mice of two groups after 18 days. (B) Tumor growth curves showed the comparison of tumor formation between the miR-33a-silenced group and the control group. ANOVA test was used for statistical analysis. ${ }^{*} \mathrm{P}<0.05$ (C) Tumor weight from the miR33a-knockdown group was significantly lower compared to that noted in the control group. t-test was used for statistical analysis. ${ }^{*} \mathrm{P}<0.05$.

mRNA of SIRT6 is a target of miR-33a in glioma cells. According to the prediction of bioinformatic software (Targetscan), SIRT6 was considered as one of the candidates with which miR-33a could bind directly (Fig. 5A). To experimentally validate the target prediction, U87 and U251 cells were transfected with miR-33a mimic or inhibitor. miR-33a mimic was shown to significantly decrease the expression of SIRT6 at both the mRNA and protein levels in the U87 cells ( $\mathrm{P}<0.05$, Fig. 5B). Consistently, miR-33a inhibitor displayed a promotive effect on the mRNA and protein levels of SIRT6 in the U251 cells $(\mathrm{P}<0.05$, Fig. 5C). More importantly, transfection of miR-33a mimic greatly attenuated the luciferase activity of the wt SIRT6-3'UTR reporter in the U87 cells while mutation in SIRT6-3'UTR evidently damaged the response of luciferase activity to miR-33a mimic ( $\mathrm{P}<0.05$, Fig. 5D). Next, the levels of SIRT6 mRNA were detected by qRT-PCR in glioma and corresponding normal tissues. Underexpression of SIRT6 mRNA was found in glioma specimens compared to that noted in the corresponding normal tissues ( $\mathrm{P}<0.05$, Fig. 5E). Spearman's correlation analysis revealed that the levels of miR-33a were inversely correlated with SIRT6 mRNA expression in the glioma tissues $(\mathrm{r}=-0.573, \mathrm{P}<0.001)$. Moreover, representative immunohistochemical staining showed that miR-33a lowexpressing tumors showed strong staining of SIRT6, while a weak signal of SIRT6 was observed in the miR-33a highexpressing cases (Fig. 6). Taken together, miR-33a strongly regulates the expression of SIRT6 by interacting with the 3'UTR region of SIRT6 mRNA in glioma cells.
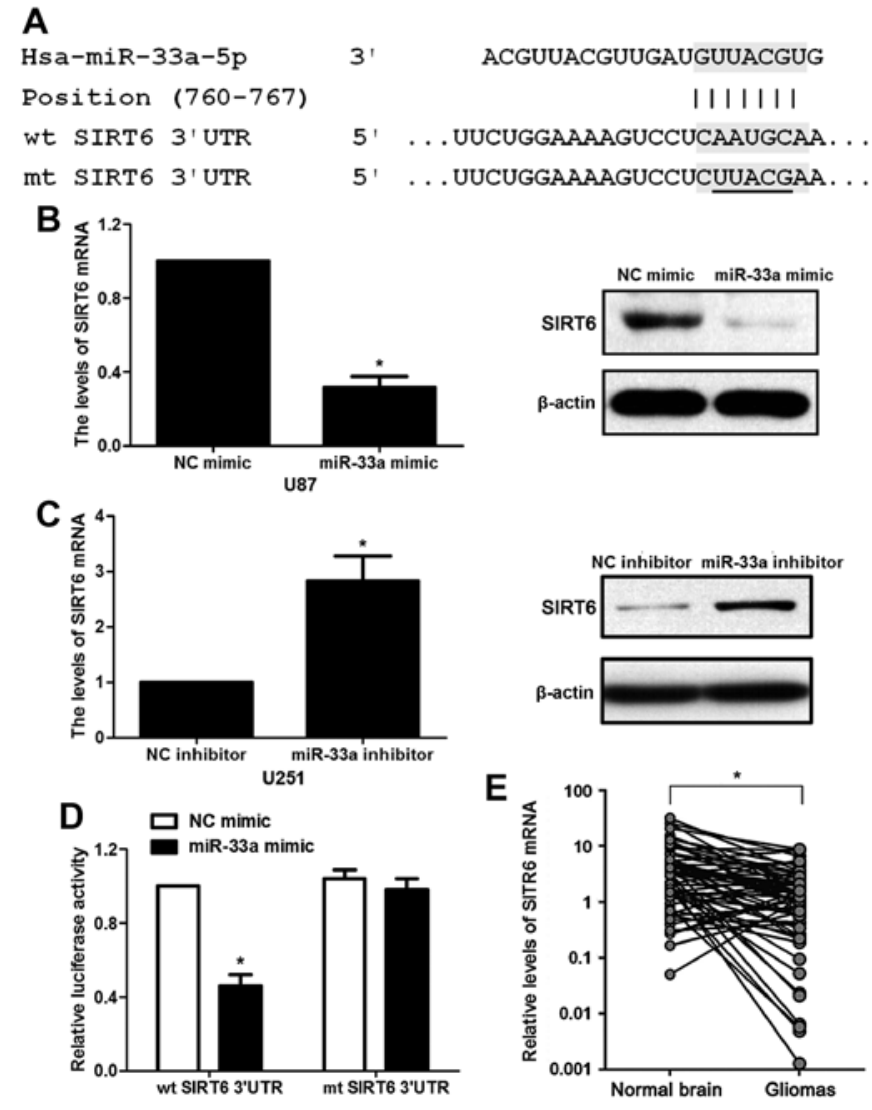

Figure 5. SIRT6 is the direct target of miR-33a in glioma cells. (A) The potential binding site of miR-33a in the wild-type (wt) 3'UTR sequence of SIRT6. The underlined part is the mutant site designed for mutant (mt) SIRT63'UTR. (B and C) The regulatory roles of miR-33a on SIRT6 expression in glioma cell lines were determined by qRT-PCR and western blot analysis. t-test was used for statistical analysis. ${ }^{~} \mathrm{P}<0.05$. (D) miR-33a overexpression notably decreased the luciferase activity of wt SIRT6-3'UTR, while showed no significant effect on the luciferase activity of mt SIRT6-3'UTR. t-test was used for statistical analysis. ${ }^{*} \mathrm{P}<0.05$. (E) qRT-PCR was performed to identify the relative expression of SIRT6 mRNA in 60 paired primary glioma samples. t-test was used for statistical analysis. " $\mathrm{P}<0.05$. SIRT6, sirtuin 6 .

SIRT6 enhances $\mathrm{H}_{2} \mathrm{O}_{2}$-induced oxidative stress and apoptosis possibly by suppressing the JAK2/STAT3 pathway. Previous research revealed that SIRT6 overexpression resulted in reduced cell survival and increased ROS production under $\mathrm{H}_{2} \mathrm{O}_{2}$-induced oxidative stress in neuronal cells (20). To disclose the potential effect of SIRT6 on $\mathrm{H}_{2} \mathrm{O}_{2}$-induced cancer cell injury, U251 cells that were transfected with pcDNA3.1-SIRT6 or empty vector were treated with $\mathrm{H}_{2} \mathrm{O}_{2}$ for $1 \mathrm{~h}$ and then cultured for $24 \mathrm{~h}$. The restoration of SIRT6 was confirmed by immunoblotting in the U251 cells $(\mathrm{P}<0.05$, Fig. 7A). Our results revealed that SIRT6 overexpression increased the levels LDH and ROS while reducing cell survival compared to the control cells ( $\mathrm{P}<0.05$, respectively, Fig. 7B-D). The Fas ligand/ Fas death receptor pathways and mitochondrial pathway are involved in ROS-induced apoptosis (22). Next, western blotting results indicated that SIRT6 overexpression upregulated the levels of Bax and cleaved caspase- 8 while downregulating Bcl-2 expression in the U251 cells (Fig. 8A). JAK2/STAT3 pathway was found to enhance cell proliferation and to prohibit apoptosis, and blocking this pathway increased the sensitivity to oxidative stress-induced cell injury (23). Our data revealed that SIRT6 restoration repressed the activation of the 


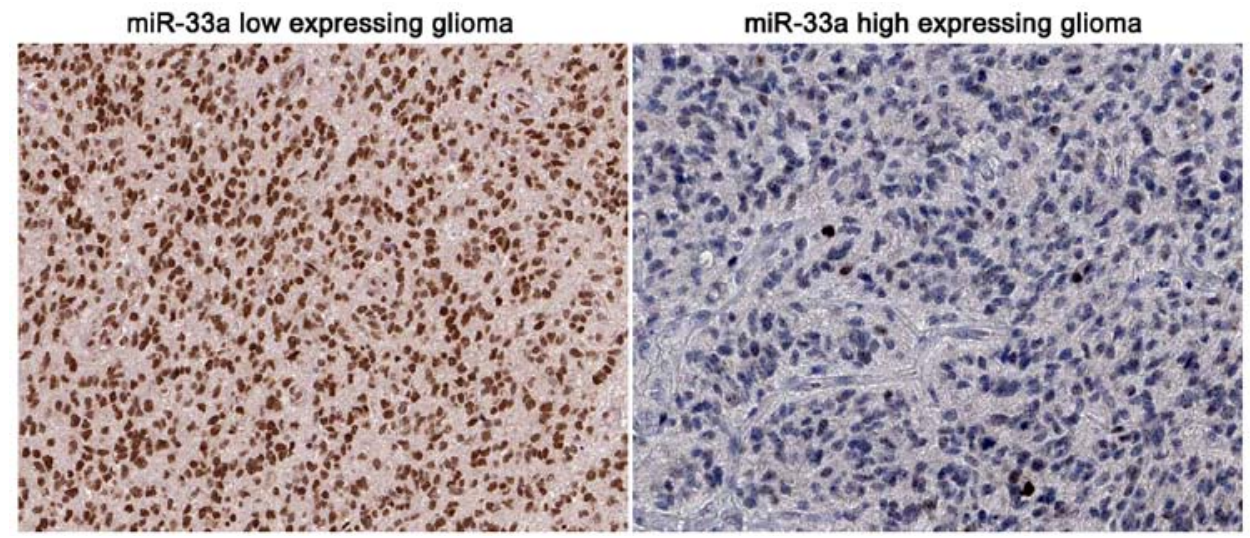

Figure 6. Immunohistochemical staining of SIRT6 in gliomas. Representative sections indicated that miR-33a low expressing gliomas showed strong staining of SIRT6, while a weak signal of SIRT6 was detected in miR-33a high expressing cases. SIRT6, sirtuin 6.
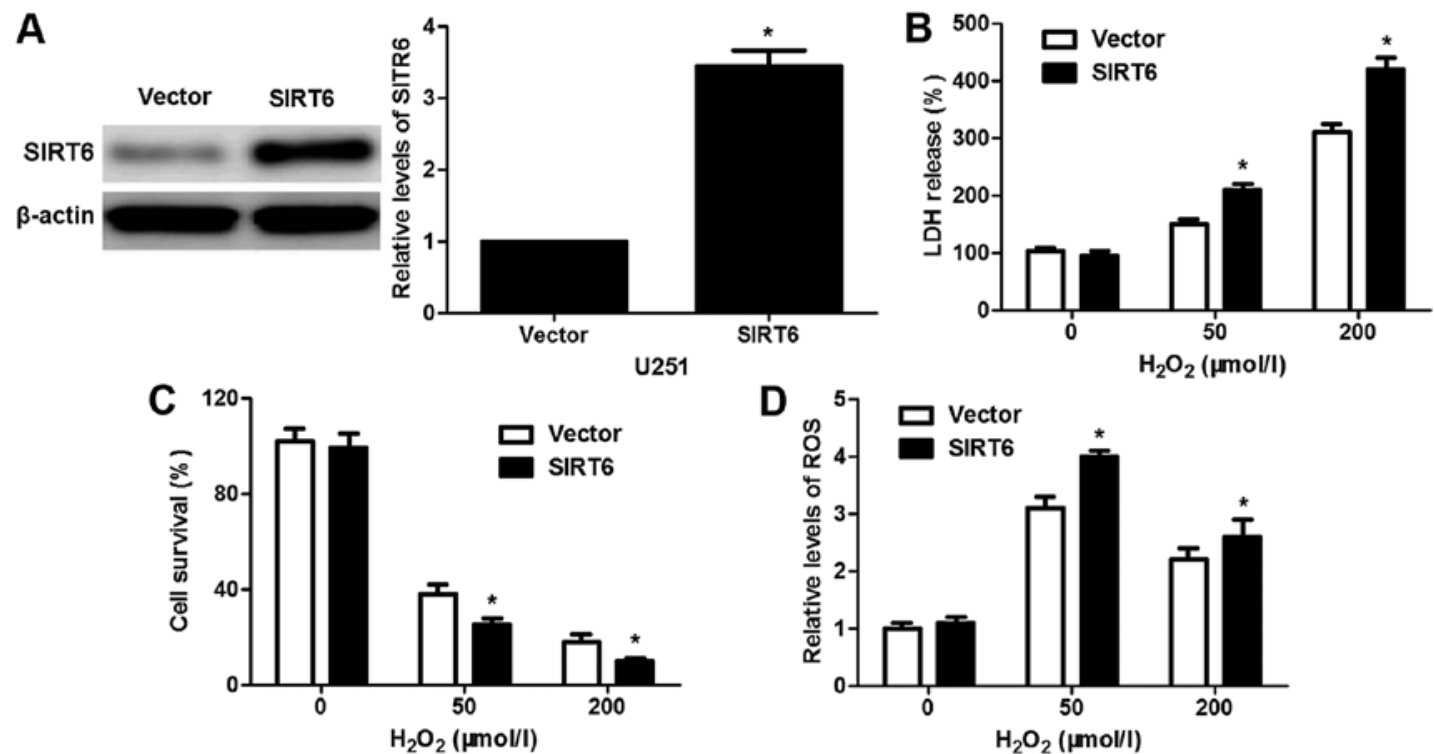

Figure 7. SIRT6 enhances $\mathrm{H}_{2} \mathrm{O}_{2}$-induced cell injury. (A) U251 cells that were transfected with pcDNA3.1-SIRT6 and empty vector, respectively, were subjected to immunoblotting for SIRT6 expression. t-test was used for statistical analysis. "P $<0.05$. (B and C) SIRT6-overexpressing U251 cells and control cells were pretreated with $\mathrm{H}_{2} \mathrm{O}_{2}$ for $1 \mathrm{~h}$ and then cultured for $24 \mathrm{~h}$. LDH release and CCK-8 assays were performed to detect cell death and viability, respectively. t-test was used for statistical analysis. " $\mathrm{P}<0.05$. (D) SIRT6-overexpressing U251 cells and control cells were pretreated with $\mathrm{H}_{2} \mathrm{O}_{2}$ for $1 \mathrm{~h}$ and then cultured for $6 \mathrm{~h}$. SIRT6 restoration increased intracellular ROS levels compared to control cells. t-test was used for statistical analysis. * $\mathrm{P}<0.05$. SIRT6, sirtuin 6; LDH, lactate dehydrogenase; ROS, reactive oxygen species.
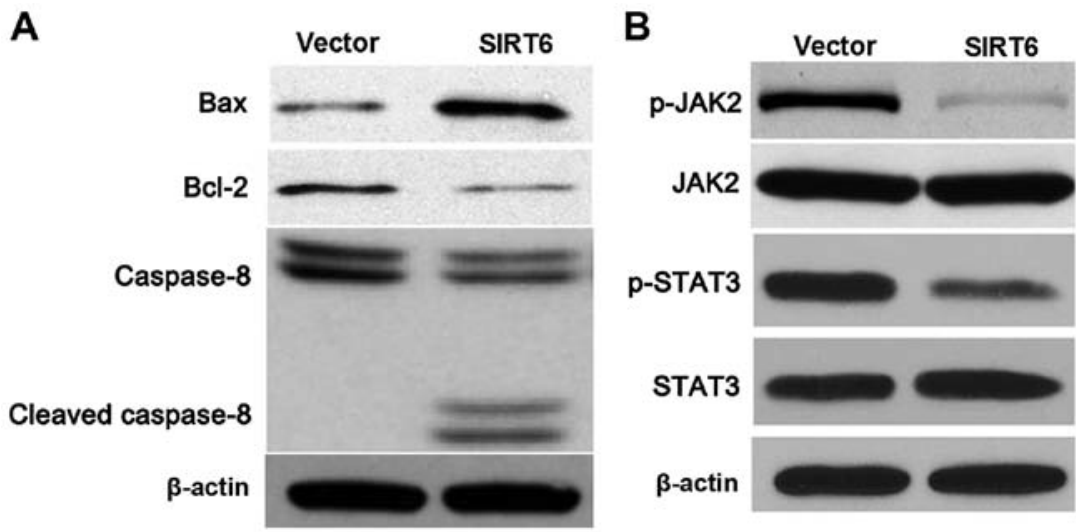

Figure 8. SIRT6 induces apoptosis and inhibits JAK2/STAT3 pathway. (A) U251 cells that were transfected with pcDNA3.1-SIRT6 and empty vector, respectively, were subjected to immunoblotting for Bax, Bcl-2 and caspase-8 expression. (B) SIRT6 overexpression prohibited the activation of JAK2/STAT3 pathway with decreased levels of phosphorylated JAK2 and STAT3 in U251 cells. SIRT6, sirtuin 6; JAK2, Janus kinase 2; STAT3, signal transducer and activator of transcription 3 . 
JAK2/STAT3 pathway with reduced levels of phosphorylated JAK2 and STAT3 in the U251 cells (Fig. 8B). Altogether, SIRT6 enhanced oxidative stress-induced cell injury and apoptosis possibly by inhibition of the JAK2/STAT3 pathway.

\section{Discussion}

miRNAs are identified as critical regulators of cancer growth and metastasis $(24,25)$. Among numerous miRNAs, miR-33a has been confirmed as a critical tumor-suppressive miRNA and a prognostic marker of human cancers including $\operatorname{NSCLC}(9,26)$ and pancreatic cancer (16), while, miR-33a exerts an oncogenic role in prostate cancer (17) and glioblastoma (19). Here, our data indicated that overexpression of miR-33a was common in glioma tissues. In addition, the levels of miR-33a were intensively elevated in glioma cell lines compared to that noted in the NHA cells. Glioma patients with a large tumor size and advanced WHO grade showed prominently higher levels of miR-33a. Furthermore, a high level of miR-33a was implicated in poor prognosis prediction, which was consistent with a previous study of glioblastoma reported by Wang et al (19). Thus, overexpression of miR-33a may be used as a potential indicator for the poor prognosis of glioma patients. The roles of miR-33a in the modulation of cell growth and self-renewal have been reported in glioma-initiating cells (19). In our recent study, loss of miR-33a reduced proliferation and increased apoptosis of U251 cells. The opposite data were obtained with miR-33a mimic treatment in U87 cells. These data reveal that miR-33a exerts its oncogenic role by regulating proliferation and apoptosis in glioma.

SIRT6 has been confirmed as an important tumor suppressor and regulates the progression and metastasis of pancreatic cancer (27). SIRT6 is post-transcriptionally modulated by miR-122 in HCC (28). A previous study reported that $\mathrm{miR}-33 \mathrm{a} / \mathrm{b}$ regulate fatty acid oxidation by targeting SIRT6 in hepatocytes (29). In this study, our data from the luciferase reporter assay, qRT-PCR and immunoblotting demonstrated that SIRT6 is a direct target of miR-33a in glioma cells. Furthermore, SIRT6 was underexpressed and was negatively correlated with miR-33a expression in the glioma specimens. In contrast, upregulation of SIRT6 was reported in NSCLC and HCC $(30,31)$, which was consistent with the downregulation of miR-33a in these cancers $(9,12)$. All these data suggest an inverse correlation between miR-33a and SIRT6 expression. SIRT6 facilitates the sensitivity of neuroblastoma cells to oxidative stress (20). Thus, we focused on the correlation between SIRT6 expression and oxidative stress-induced cell injury in glioma. Notably, SIRT6 restoration increased the levels of LDH and ROS and reduced cell survival under $\mathrm{H}_{2} \mathrm{O}_{2}$ treatment in glioma cells. A previous study reported that SIRT6 restrained the growth of glioma cells by inducing apoptosis and repressing the JAK2/STAT3 pathway (23). Here, we showed that SIRT6 induced apoptosis with increased levels of Bax and cleaved caspase- 8 , and decreased expression of $\mathrm{Bcl}-2$ in glioma cells. Researchers report that ROS activate caspase- 8 to induce apoptosis. In addition, ROS increase the ratio of $\mathrm{Bax} / \mathrm{Bcl}-2$ via downregulation of Bcl-2 and upregulation of Bax and subsequently participate in the mitochondrial pathways of apoptosis. Furthermore, our data disclosed that SIRT6 overexpression reduced the activation of the JAK2/STAT3 pathway, which functions in an oxidative stress resistant role by promoting proliferation and repressing apoptosis. Taken together, SIRT6 facilitates oxidative stress-induced growth arrest and apoptosis by repressing the JAK2/STAT3 pathway in glioma.

In summary, our findings highlight a miR-33a/SIRT6 pathway, the dysregulation of which leads to the proliferation and apoptotic resistance of glioma. Furthermore, our study provides a detailed insight into the mechanism underlying the role of SIRT6 in glioma. Thus, more research is needed to explore the potential of miR-33a and SIRT6 as novel therapeutic targets for the treatment of glioma.

\section{Acknowledgements}

This study was supported by the Natural Science Basic Research Plan in Shaanxi Province of China (program no. 2014JM4139).

\section{References}

1. Awad AJ, Burns TC, Zhang Y and Abounader R: Targeting MET for glioma therapy. Neurosurg Focus 37: E10, 2014.

2. Katsetos CD, Reginato MJ, Baas PW, D'Agostino L, Legido A, Tuszyn Ski JA, Dráberová E and Dráber P: Emerging microtubule targets in glioma therapy. Semin Pediatr Neurol 22: 49-72, 2015.

3. Klein M: Treatment options and neurocognitive outcome in patients with diffuse low-grade glioma. J Neurosurg Sci 59: 383-392, 2015.

4. Kang JH and Adamson C: Novel chemotherapeutics and other therapies for treating high-grade glioma. Expert Opin Investig Drugs 24: 1361-1379, 2015.

5. Brandner S and von Deimling A: Diagnostic, prognostic and predictive relevance of molecular markers in gliomas. Neuropathol Appl Neurobiol 41: 694-720, 2015.

6. Wang BC and Ma J: Role of microRNAs in malignant glioma. Chin Med J (Engl) 128: 1238-1244, 2015.

7. Garzon R, Calin GA and Croce CM: MicroRNAs in cancer. Annu Rev Med 60: 167-179, 2009.

8. Thomas M, Lange-Grünweller K, Weirauch U, Gutsch D, Aigner A, Grünweller A and Hartmann RK: The proto-oncogene Pim-1 is a target of miR-33a. Oncogene 31: 918-928, 2012.

9. Yang L, Yang J, Li J, Shen X, Le Y, Zhou C, Wang S, Zhang S, Xu D and Gong Z: MircoRNA-33a inhibits epithelial-to-mesenchymal transition and metastasis and could be a prognostic marker in non-small cell lung cancer. Sci Rep 5: 13677, 2015.

10. Du M, Zhang Y, Mao Y, Mou J, Zhao J, Xue Q, Wang D, Huang J, Gao S and Gao Y: miR-33a suppresses proliferation of NSCLC cells via targeting METTL3 mRNA. Biochem Biophys Res Commun 482: 582-589, 2017.

11. Zhang M, Gong W, Zuo B, Chu B, Tang Z, Zhang Y, Yang Y, Zhou D, Weng M, Qin Y, et al: The microRNA miR-33a suppresses IL-6-induced tumor progression by binding Twist in gallbladder cancer. Oncotarget 7: 78640-78652, 2016.

12. Han SY, Han HB, Tian XY, Sun H, Xue D, Zhao C, Jiang ST, He XR, Zheng WX, Wang J, et al: MicroRNA-33a-3p suppresses cell migration and invasion by directly targeting PBX3 in human hepatocellular carcinoma. Oncotarget 7: 42461-42473, 2016.

13. Zhang C, Zhang Y, Ding W, Lin Y, Huang Z and Luo Q: miR-33a suppresses breast cancer cell proliferation and metastasis by targeting ADAM9 and ROS1. Protein Cell 6: 881-889, 2015.

14. Zhou J, Xu D, Xie H, Tang J, Liu R, Li J, Wang S, Chen X, Su J, Zhou X, et al: miR-33a functions as a tumor suppressor in melanoma by targeting HIF-1 $\alpha$. Cancer Biol Ther 16: 846-855, 2015.

15. Wolfe AR, Bambhroliya A, Reddy JP, Debeb BG, Huo L, Larson R, Li L, Ueno NT and Woodward WA: miR-33a decreases high-density lipoprotein-induced radiation sensitivity in breast cancer. Int J Radiat Oncol Biol Phys 95: 791-799, 2016.

16. Liang C, Yu XJ, Guo XZ, Sun MH, Wang Z, Song Y, Ni QX, Li HY, Mukaida N and Li YY: MicroRNA-33a-mediated downregulation of Pim-3 kinase expression renders human pancreatic cancer cells sensitivity to gemcitabine. Oncotarget 6: 14440-14455, 2015. 
17. Li Q, Lu S, Li X, Hou G, Yan L, Zhang W and Qiao B: Biological function and mechanism of miR-33a in prostate cancer survival and metastasis: via downregulating Engrailed-2. Clin Transl Oncol 19: 562-570, 2017.

18. Zhou Y, Huang Z, Wu S, Zang X, Liu M and Shi J: miR-33a is up-regulated in chemoresistant osteosarcoma and promotes osteosarcoma cell resistance to cisplatin by down-regulating TWIST. J Exp Clin Cancer Res 33: 12, 2014.

19. Wang H, Sun T, Hu J, Zhang R, Rao Y, Wang S, Chen R, McLendon RE, Friedman AH, Keir ST, et al: miR-33a promotes glioma-initiating cell self-renewal via PKA and NOTCH pathways. J Clin Invest 124: 4489-4502, 2014.

20. Shao J, Yang X, Liu T, Zhang T, Xie QR and Xia W: Autophagy induction by SIRT6 is involved in oxidative stress-induced neuronal damage. Protein Cell 7: 281-290, 2016.

21. Song J, Ke SF, Zhou CC, Zhang SL, Guan YF, Xu TY, Sheng CQ, Wang P and Miao CY: Nicotinamide phosphoribosyltransferase is required for the calorie restriction-mediated improvements in oxidative stress, mitochondrial biogenesis, and metabolic adaptation. J Gerontol A Biol Sci Med Sci 69: 44-57, 2014

22. Jeong $\mathrm{CH}$ and Joo SH: Downregulation of reactive oxygen species in apoptosis. J Cancer Prev 21: 13-20, 2016.

23. Feng J, Yan PF, Zhao HY, Zhang FC, Zhao WH and Feng M: SIRT6 suppresses glioma cell growth via induction of apoptosis inhibition of oxidative stress and suppression of JAK2/STAT3 signaling pathway activation. Oncol Rep 35: 1395-1402, 2016.

24. Jansson MD and Lund AH: MicroRNA and cancer. Mol Oncol 6: 590-610, 2012
25. Nugent M: microRNA and Bone Cancer. Adv Exp Med Biol 889 201-230, 2015.

26. Kuo PL, Liao SH, Hung JY, Huang MS and Hsu YL: MicroRNA-33a functions as a bone metastasis suppressor in lung cancer by targeting parathyroid hormone related protein. Biochim Biophys Acta 1830: 3756-3766, 2013.

27. Kugel S, Sebastián C, Fitamant J, Ross KN, Saha SK, Jain E, Gladden A, Arora KS, Kato Y, Rivera MN, et al: SIRT6 suppresses pancreatic cancer through control of Lin28b. Cell 165: 1401-1415, 2016.

28. Elhanati S, Ben-Hamo R, Kanfi Y, Varvak A, Glazz R, Lerrer B, Efroni S and Cohen HY: Reciprocal regulation between SIRT6 and miR-122 controls liver metabolism and predicts hepatocarcinoma prognosis. Cell Reports 14: 234-242, 2016.

29. Dávalos A, Goedeke L, Smibert P, Ramírez CM, Warrier NP, Andreo U, Cirera-Salinas D, Rayner K, Suresh U, PastorPareja JC, et al: $\mathrm{miR}-33 \mathrm{a} / \mathrm{b}$ contribute to the regulation of fatty acid metabolism and insulin signaling. Proc Natl Acad Sci USA 108: 9232-9237, 2011

30. Lee N, Ryu HG, Kwon JH, Kim DK, Kim SR, Wang HJ, Kim KT and Choi KY: SIRT6 depletion suppresses tumor growth by promoting cellular senescence induced by DNA damage in HCC. PLoS One 11: e0165835, 2016.

31. Bai L, Lin G, Sun L, Liu Y, Huang X, Cao C, Guo Y and Xie C: Upregulation of SIRT6 predicts poor prognosis and promotes metastasis of non-small cell lung cancer via the ERK1/2/MMP9 pathway. Oncotarget 7: 40377-40386, 2016. 